\title{
Importancia del manejo de competencias tecnológicas en las prácticas docentes de la Universidad Nacional Experimental de la Seguridad (UNES)
}

\author{
Cruz Rodríguez, Eglis Del Carmen \\ Importancia del manejo de competencias tecnológicas en las prácticas docentes de la Universidad \\ Nacional Experimental de la Seguridad (UNES) \\ Revista Educación, vol. 43, núm. 1, 2019 \\ Universidad de Costa Rica, Costa Rica \\ Disponible en: http://www.redalyc.org/articulo.oa?id=44057415013 \\ DOI: https://doi.org/10.15517/revedu.v43i1.27120
}

Esta obra está bajo una Licencia Creative Commons Atribución-NoComercial-SinDerivar 3.0 Internacional. 
Importancia del manejo de competencias tecnológicas en las prácticas docentes de la Universidad Nacional Experimental de la Seguridad (UNES)

\author{
The importance of digital skills in teacher training at the National Experimental Security University (UNES) in \\ Venezuela \\ Eglis Del Carmen Cruz Rodriguez \\ Universidad Nacional Experimental de la Seguridad, \\ Venezuela \\ despacho@unes.edu.ve \\ DOI: https://doi.org/10.15517/revedu.v43i1.27120 \\ Redalyc: http://www.redalyc.org/articulo.oa?id=44057415013
}

iD http://orcid.org/0000-0003-0050-0649

Recepción: 19 Noviembre 2016

Aprobación: 25 Noviembre 2018

\title{
RESUMEN:
}

La presente investigación trata sobre la importancia del manejo de las competencias tecnológicas digitales en las prácticas docentes, es de carácter cualitativo, para lo cual se efectuó un estudio de caso en el que se aplicó una encuesta con un índice de confiabilidad $(0,75)$ muy alto según la escala Alpha de Cronbach, esta se aplicó para medir el nivel de destrezas que poseen los docentes de la Universidad Nacional Experimental de la Seguridad en competencias digitales, en sus ambientes educativos. Estuvo constituida por una muestra de 49 facilitadores, los resultados permitieron corroborar que un sector de los docentes no poseen los conocimientos básicos y en su mayoría se evidencia fuertes debilidades en el desarrollo apropiado de las competencias digitales, un índice elevado de la población en estudio manifiesta un nivel de rechazo en el manejo de la tecnología como estrategias pedagógicas y en líneas generales una población representativa no alcanza las competencias técnicas digitales, manifestado en un $37,3 \%$ el cual no ha participado en talleres de alfabetización tecnológica. Se limitan al uso de herramientas digitales clásicas como el Power Point. Entre las recomendaciones se propone un plan de capacitación al personal docente en el uso de herramientas digitales, diseño de software educativo, entre otros, con el objetivo de actualizar en el uso de estrategias pedagógicas que permitan el uso efectivo del internet en los ambientes educativos.

Palabras Clave: Competencias tecnológicas digitales, habilidades, Tecnología de la Información y la Comunicación (TIC), estrategias de aprendizajes, actitud, manejo, herramientas, software educativo, iniciación informática, nuevas tecnologías.

\section{Abstract:}

This study addresses the importance of digital literacy and developing digital technology skills among teachers. This is a qualitative case study based on a digital skills survey conducted among the faculty of the National Experimental Security University (UNES) in Venezuela. The survey was scored according to the Cronbach Alpha Scale and had a reliability index of 0.75. The objective of the survey was to determine the digital competencies and application of these competencies in the classroom by UNES teaching staff. The results of this study allowed us to confirm that most of the UNES teaching staff studied lack basic digital knowledge and showed strong weaknesses in appropriately developing their digital skills. A high percentage of the population group studied expressed a certain level of rejection of the learning curve required to embrace technology as part of their pedagogical strategy. Overall, 37.3\% of the representative population stated that they lacked basic digital skills, had never received any digital oriented training and were only versed in using more traditional software such as Power Point. Among the different recommendations made was to plan teacher training workshops in the use of educational software and educational software design, for example, to be update teaching skills required for effective use of the Internet in educational settings.

KEYWORDS: Digital technology competencies and skills, Information and Communication Technology (ICT), learning strategies, attitude, management, tools, educational software, computer initiation, new technologies, digital literacy, digital learning curve .

\section{INTRODUCCIÓN}

Las universidades como centro de estudio están sujetas a las dinámicas educativas impulsando entre sus tareas fundamentales la producción de conocimientos, por lo cual la formación y preparación de sus estudiantes al 
servicio de una sociedad más competitiva y desarrollada es una meta impostergable. En el recinto universitario los procesos de enseñanza deberán estar ajustados a las innovaciones tecnológicas, de forma tal que el personal docente esté lo suficientemente capacitado y actualizado en las Tecnologías de la Información y la Comunicación (TIC), en este sentido las instituciones educativas según sus particularidades culturales, económicas, tecnológicas, entre otras, han asumido reformas educativas para su integración a las nuevas tecnologías.

Entonces resulta importante enfatizar el rol del docente en su desempeño en aras de optimizar su función principal como facilitador de procesos de aprendizajes, para lo cual sus estrategias y recursos pedagógicos deberán están basados en la apropiación e integración de las herramientas digitales que ofrecen el uso de las TIC, en la planificación educativa. En diseño y producción de TIC para la formación, según Cabero y Romero (2010):

La enseñanza deja de ser un problema de profesores y alumnos en su clase. Por lo tanto podemos decir que las TIC vienen a exigir y a facilitar una visión más exigente y amplia de la profesionalidad de los docentes, exigiendo para su integración de mayores dosis de planificación y trabajo. Intentando salvar la mera integración de tecnologías en los centros educativos y propiciando verdaderos procesos de integración curricular de las TIC. (párr.1)

En lo referido anteriormente, la sociedad del conocimiento establece nuevos métodos de enseñanza dejando a un lado las formas tradicionales de educación basadas en el uso de la memoria. En este terreno los entornos de aprendizajes modernos dinamizan la escala de aprendizajes que proporciona la interactividad online, acompañada esta de la selección de información apropiada, y sus contraste con diferentes puntos de vista transdisciplinario, construyendo una red virtual de intercambio constante con múltiples participantes del proceso educativo, en la cual el binomio docente-estudiantes debe estar preparado ante el reto que significa producción de saberes. Al respecto Heras, Roa y Espinoza (2015) comentan:

En la actualidad las tecnologías de la Información y la Comunicación (TIC) en el contexto educativo son objeto de revisión debido a la importancia que señala que un profesor tenga las competencias necesarias para hacer frente a este cambio tecnológico, es decir, esté capacitado para su uso, manejo y posterior implementación didáctica en el aula, esto supone ser competente, ser un docente del siglo XXI. (p. 3)

En la actualidad las Tecnologías de la Información y la Comunicación (TIC) en el contexto educativo son objeto de revisión debido a la importancia que señala que un docente tenga las competencias necesarias para hacer frente a este cambio tecnológico, es decir, esté capacitado para su uso, manejo y posterior implementación didáctica en el aula, esto supone ser competente, ser un docente del siglo XXI.

En este marco de ideas de los docentes necesitan de una profunda transformación en lo que corresponde a la actualización profesional en el siglo XXI, específicamente en el campo de las Tecnologías de la Información y la Comunicación (TIC) para el apropiado manejo de tecnologías digitales. Por lo cual en la presente investigación se consideró de importancia efectuar un estudio de caso, en el cual se precisó cuáles son las competencias tecnológicas en las TIC que poseen los educadores de la Universidad Nacional Experimental de la Seguridad. Al respecto para definir las competencias se hace necesario recurrir a diferentes visiones. Las competencias se definen como las destrezas habilidades y actitudes que permiten un buen desempeño en la docencia. En Sánchez y Talavera (2008):

\footnotetext{
e entenderá por competencia el buen desempeño en contextos diversos y auténticos basado en la integración y activación de conocimientos, técnicas, procedimientos, habilidades y destrezas, actitudes y valores. Se entiende también como el conjunto de saberes que involucran conocimientos, acciones, sentido axiológico y propósitos que desarrolla la persona tanto en el contexto de su formación, en el ejercicio profesional como en su vida personal y social, que combinados, coordinados e integrados, facilitan su actuación eficazmente. (pág. 9)
}

En lo que se refiere a las habilidades cognitivas en el manejo de tecnologías de la información y la comunicación en la formación de los docentes, los autores Arrufat y Sánchez (2010), indican: 
Las competencias digitales se han asociado a dos objetivos clave de la preparación del futuro docente: por un lado, conocer y reflexionar sobre el contexto tecnológico en el que se desenvuelven sus alumnos y, por otro, desarrollar nuevas habilidades que les permitan utilizar las tecnologías para favorecer aprendizajes significativos. (pág. 3)

Otro enfoque del concepto de competencia digital es el publicado en el Real Decreto Boletín Oficial del Estado (BOE) España n 1513 (2006, citado por los autores González y Wagenaar (2003), el cual se refiere al tratamiento de la información de la siguiente forma:

Esta competencia consiste en disponer de habilidades para buscar, obtener, procesar y comunicar información, y para transformarla en conocimiento. Incorpora diferentes habilidades, que va desde el acceso a la información hasta su transmisión en distintos soportes una vez tratada, incluyendo la utilización de las tecnologías de la información y la comunicación como elemento esencial para informarse, aprender y comunicarse [...] (p.17).

Los temas tratados en el párrafo anterior permiten acotar, en el siglo XXI era de la información y comunicación, las universidades son garantes de la transformación educativa partiendo de los nuevos modelos de construcción del conocimiento, desde la perspectiva crítica basados en las innovaciones tecnológicas, según los objetivos planteados acorde a las particularidades de cada proceso según el contexto educativo. Considerando así el aprendizaje como eje fundamental en la educación, el rol del docente consiste en facilitar dichos procesos por lo cual deberá poseer las competencias necesarias que permitan alcanzar las metas propuestas en la formación estudiantil. Páez (2008), indica:

La sociedad de la información está cambiando ese paradigma instruccional. Los métodos de enseñanza y muchos programas de instrucción basados en el computador han cambiado la visión sobre el aprendizaje. Se está pasando de la pedagogía de la adquisición del saber a una pedagogía del aprendizaje de procesos. Ahora el aprendizaje se representa por un grupo de participantes discutiendo, interactuando, aprendiendo unos de otros, con el profesor como otro miembro, construyendo respuestas a partir de sus propias experiencias, de la lluvia de ideas y de debates críticos, influyendo unos sobre otros, produciendo un aprendizaje social, activo, constructivo, interactivo, colaborativo. (párr. 5)

Considerando las repercusiones originadas por la implementación de las TIC en los modelos educativos actuales, se hace especial énfasis en impulsar los cambios de paradigmas, al respecto el docente deberá ser un facilitador con las cualidades de motivar e incentivar el uso apropiado y efectivo de las herramientas informáticas, con el objetivo de desarrollar en el proceso de enseñanza aprendizaje una actividad permanente de actualización en el uso de las nuevas tecnologías. En 1997, Adell comenta:

Los nuevos entornos de enseñanza/aprendizaje exigen nuevos roles en profesores y estudiantes. La perspectiva tradicional en educación superior, por ejemplo, del profesor como única fuente de información y sabiduría y de los estudiantes como receptores pasivos debe dar paso a papeles bastante diferentes. (párr. 1)

Si bien el uso de las TIC es una herramienta indispensable para desarrollar las potencialidades de la praxis docente, ampliando las posibilidades de la interacción educativa. El profesional docente que alcance las competencias tecnológicas tenderá a transformar las formas tradicionales de enseñanza que hasta el momento se han utilizado, generando cambios de estrategias didácticas efectivas y novedosas. Entre ellos se mencionan el tema de los espacios de aprendizajes asincrónicos, los cuales emergen en el contexto novedoso de permitirles a los participantes en proceso de formación de disfrutar la oportunidad de asistir a las aulas virtuales, las cuales garantizan el acceso a los estudios para la adquisición de habilidades y destrezas sin limitaciones espaciostemporal. Por lo cual el manejo de la tecnología aunado a las estrategias de organización y planificación de las actividades, selección adecuada y destacada de la información para el desarrollo de un tema de estudio, integrado a la creatividad e innovación del docente son los elementos que facilitaran a los estudiantes aproximarse a la resolución de problemas derivados de la realidad. "Las habilidades consideradas como centrales en el desarrollo de los sujetos ante la sociedad del conocimiento. Las cuales son: 1. Tratamiento de la información 2. Intercambio de información y conocimiento y 3. Construcción de conocimiento y solución de problemas”. (González, 2018, párr. 53) 
Al respecto El Proyecto Estándares UNESCO de Competencia en TIC para Docentes (UNESCO, 2008), establece los parámetros necesarios que se deben dinamizar para garantizar la formación del docente en las nuevas tecnologías, al respecto se comenta:

Los docentes necesitan estar preparados para empoderar a los estudiantes con las ventajas que les aportan las TIC. Escuelas y aulas -ya sean presenciales o virtuales- deben contar con docentes que posean las competencias y los recursos necesarios en materia de TIC y que puedan enseñar de manera eficaz las asignaturas exigidas, integrando al mismo tiempo en su enseñanza conceptos y habilidades de estas. Las simulaciones interactivas, los recursos educativos digitales y abiertos (REA) los instrumentos sofisticados de recolección y análisis de datos son algunos de los muchos recursos que permiten a los docentes ofrecer a sus estudiantes posibilidades, antes inimaginables, para asimilar conceptos. (p. 2)

Lo expuesto anteriormente confirma que los medios instruccionales o recursos pedagógicos de la acción educativa en la era de la información han originado una revolución en los métodos tradicionales de la planificación educativa, dando paso al uso sofisticado de múltiples herramientas digitales para el diseño instruccional fundamentado en las formas interactivas; ampliando así las posibilidades del aprendizaje colaborativo, en el cual el estudiante se involucra de forma activa y se retroalimenta en un entorno participativo, desarrollando las destreza y habilidades que le permiten alcanzar los objetivos propuestos. Desde esta perspectiva es importante determinar las diferencias de los términos nuevas tecnologías aplicadas a la educación al de tecnología de la educación, según Cacheiro (2014) comenta:

La Tecnología de la Educación se centra en estudiar las estrategias de enseñanza de carácter multimedia, mientras que las Nuevas Tecnologías aplicadas a la Educación pretenden la capacitación del futuro profesor como usuario de recursos multimedia. En el primero el foco se centra más en el diseño de recursos tecnológicos para el proceso de enseñanzaaprendizaje, mientras que en el segundo trata de adentrarse en su utilización dentro del proceso anteriormente mencionado. (párr. 14)

En este mismo orden de ideas la relevancia de capacitar a los docentes en el manejo de la plataforma Moodle, la cual se constituye en una herramienta novedosa para complementar el aprendizaje presencial, incentiva la cooperación interactiva entre estudiantes, reflexión crítica. Esta posee una interfaz de navegación eficiente en un entorno de aprendizaje dinámico y sencillo. En este punto se enfatiza que promover el uso de este tipo de herramienta como recurso de aprendizaje garantiza la actualización en el campo docente. Al respecto Mirabal (2013) comenta:

Existen numerosos ejemplos de investigaciones realizadas para documentar las prácticas docentes con Moodle. Los estudios abarcan prácticamente todos los niveles de educación y se orientan hacia diversas áreas como: innovación en la práctica docente como fruto del aprovechamiento de sus potencialidades, actitudes de estudiantes hacia las clases cuyos docentes hacen uso de esta plataforma, y utilización de Moodle como herramienta para proyectos de formación docente, entre otros. (p.39)

Las ideas expuestas anteriormente enfocan a la tecnología educativa en la era digital como un factor que origina un cambio trascendental en los medios de enseñanza, de aquí la importancia del uso de instrumentos pedagógicos basados en las nuevas tecnologías, específicamente el software educativo el cual facilita los objetivos de enseñanza y aprendizaje de forma dinámica. Al respecto en Estándares en Competencias TIC para Docentes (UNESCO, 2008), se señala que el docente deberá poseer destrezas en la materia mencionada, en este sentido se indica:

Mostrar la utilización de software de presentación multimedia y otros recursos informáticos para complementar un curso magistral; suministrar una serie de ejemplos de presentaciones multimedia educativas; solicitar a los participantes que elaboren un proyecto de clase que incluya la utilización del presentador multimedia; y pedirles que utilicen este software para preparar una presentación. (p. 20)

También, aparte de lo atinente a los procesos de actualización tecnológica del personal docente, que este tenga la capacidad de estimular en el estudiante el uso apropiado y crítico de las herramientas TIC, considerando la importancia a la iniciación informática o alfabetización tecnológica, acompañado de la 
dotación de los elementos tecnológicos tales como acceso a internet, computadoras, dispositivos móviles, entre otros que faciliten el acceso a la WEB 2.0 dinamizando los niveles de aprendizajes al respecto, Barroso (2007) estableció, "la educación se encuentra ante grandes desafíos, que se debe empezar con procesos de alfabetización para formar a los ciudadanos competentes para vivir y trabajar en la sociedad de la información y del conocimiento, según sean las necesidades del contexto". (p. 3 )

En lo que corresponde al conjunto de destrezas digitales que debe poseer el docente, según Cabero (1999), Tejada (1999) y Majó y Marqués (2000) (citados por Marqués, 2000) resumen así las competencias en TIC que deben reunir los docentes de la sociedad de la información:

Tener una actitud positiva hacia las TIC, instrumento de nuestra cultura que conviene saber utilizar y aplicar en muchas actividades domésticas y laborales. - Conocer los usos de las TIC en el ámbito educativo. - Conocer el uso de las TIC en el campo de su área de conocimiento. - Utilizar con destreza las TIC en sus actividades: editor de textos, correo electrónico, navegación por Internet. - Adquirir el hábito de planificar el currículum integrando las TIC (como medio instrumental en el marco de las actividades propias de su área de conocimiento, como medio didáctico, como mediador para el desarrollo cognitivo). - Proponer actividades formativas a los alumnos que consideren el uso de TIC. - Evaluar el uso de las TIC. (p. 9)

Además de integrar el conjunto habilidades, actitudes y destrezas expuestas en el manejo de las TIC, Gros, Silva, Garrido, y Rodríguez (2006) citan lo siguiente:

Los futuros docentes demuestran un dominio de las competencias asociadas al conocimiento general de las TIC y el manejo de las herramientas de productividad (procesador de texto, hoja de cálculo, presentador) e Internet, desarrollando habilidades y destrezas para el aprendizaje permanente de nuevos hardware y software. (p. 21)

Por otro lado, en relación con el estudio de caso efectuado; se planteó como objetivo general de la investigación, describir el nivel de manejo que posee en competencias tecnológicas digitales el personal docente de la universidad Nacional Experimental de la Seguridad (UNES). El primer objetivo específico, es diagnosticar los conocimientos en competencias tecnológicas digitales el personal docente de la UNES y el segundo objetivo específico es evaluar el uso de recursos didácticos digitales en las prácticas docentes.

\section{Formulación del problema}

Los docentes además de adquirir los conocimientos propios de sus áreas deberán alcanzar un nivel óptimo en competencias digitales, el uso de las TIC aplicadas en la educación originando aprendizajes colaborativos y significativos para lo cual se ha de enfatizar la importancia de impartir un plan de formación en alfabetización tecnológica dirigido a los docentes UNES, considerando las actuales tendencias que facilitan acceso general del educando a las múltiples aplicaciones móviles, dispositivos y tabletas, entre otros. Por lo cual se considera necesario que los docentes manejen de forma apropiada las herramientas tecnológicas considerando los nuevos cambios y avances en la informática.

En otro orden de ideas se debe reflexionar en torno al uso adecuado de las TIC como herramienta pedagógica, orientada al desarrollo de estrategias didácticas que propicien la participación activa del estudiante; al respecto se señala en Gallego, Gámiz y Gutiérrez, (2010) “Competencia digital clave del futuro profesorado, entendida como capacidad de realizar actos profesionales con resultados reconocibles en el mundo de la profesión docente, es saber utilizar e incorporar adecuadamente en las actividades de enseñanzaaprendizaje las TIC”. (p. 3)

En concordancia a lo señalado anteriormente, el nivel de profesionalismo entendido desde la visión comprometida y ética ante el uso crítico de las fuentes digitales, su importancia y característica adecuadas al objetivo pedagógico previsto en la planificación, evitando así la pérdida de tiempo y acceso a páginas Web desactualizadas, entre otros. En este sentido, en el 2009 Tejada indica: 
La formación del profesorado no puede reducirse a la adquisición de competencias digitales o destrezas tecnológicas per se sino que debe basarse en su aplicación didáctica. Con carácter general el docente ha de planificar, impartir, tutorizar y evaluar acciones formativas, elaborando y utilizando medios y recursos didácticos, promoviendo la calidad de la formación y la actualización didáctica. (p. 9)

Sobre la base de las ideas expuestas es de suma importancia que los docentes estén al día en el manejo de las herramientas digitales, lo cual le permitiría disponer de estrategias pedagógicas que permitan incentivar la motivación de los jóvenes en la adquisición de las más recientes innovaciones informáticas, lo cual desde la dimensión del aprendizaje interactivo es un atractivo para la población estudiantil. Al respecto comenta Gómez (2010) lo siguiente:

Se observa como los profesores se encasillan en ciertos instrumentos que ya no son novedad para los alumnos, por ejemplo presentaciones en PowerPoint, búsqueda de información en internet, uso de software planos sin mayor interactividad o donde el resultado es entregado de forma inmediata y no deja tiempo a pensar. (párr. 3)

De acuerdo con lo señalado es un factor determinante las políticas institucionales que impulsen y acompañen a su personal docente en los necesarios e impostergables programas de capacitación pedagógica, que permitan elevar el nivel de manejo de herramientas digitales las cuales en un contexto amplio deberán garantizar los procesos de aprendizajes apoyados en el uso efectivo de las nociones básicas de la tecnología digital, entre ellas el uso de plataformas educativas y su relación con el reforzamiento de cambios en el entorno educativo .

\section{Fundamentación teórica}

Las innovaciones tecnológicas en el campo de la educación abren parte de las competencias específicas en un área determinada de los profesionales de la docencia, las competencias digitales desarrolladas en el manejo de las TIC, como herramientas para el desempeño adecuado de la docencia basada en estrategias innovadoras que faciliten los procesos de enseñanza y aprendizaje.

De acuerdo con lo indicado anteriormente la presente investigación está basada en gran parte en los principios establecidos en el documento titulado Estándares de Competencias en TIC para Docentes (UNESCO, 2008). En el cual se hace mención a las especificaciones de los elementos teóricos y prácticos relacionados con las competencias tecnológicas que debe poseer y alcanzar un docente para su adecuado desenvolvimiento profesional, generando la apropiación de las herramientas tecnológicas por parte de los estudiantes. Al respecto se estableció en las normas referidas en UNESCO (2008): "Los docentes deben saber dónde, cuándo (también cuándo no) y cómo utilizar la tecnología digital (TIC) en actividades y presentaciones efectuadas en el aula”. (p.15)

Atendiendo a estas consideraciones se enfatiza la importancia de un manejo amplio de las múltiples posibilidades, que le permite al docente de forma integral ejecutar una planificación educativa, respecto a los entornos de aprendizajes fundamentados en los medios de enseñanzas digitales, incorporándolos a su vez en actividades dinámicas, pedagógicas y lúdicas que hacen más atractivos e interesantes los contenidos didácticos que se traducen en el uso de nuevas tecnologías educativas. En este sentido Estándares en Competencias en TIC para Docentes (UNESCO, 2008) se

Las competencias del docente relativas al enfoque nociones básicas de TIC comprenden: competencias básicas en TIC así como la capacidad para seleccionar y utilizar métodos educativos apropiados ya existentes, juegos, entrenamiento y práctica, y contenidos de Internet en laboratorios de informática o en aulas con recursos limitados para complementar estándares de objetivos curriculares, enfoques de evaluación, unidades curriculares o núcleos temáticos y métodos didácticos. Los docentes también deben estar en capacidad de usar las TIC para gestionar datos de la clase y apoyar su propio desarrollo profesional. (p. 12) 
$\mathrm{Al}$ mismo tiempo las nociones básicas de las TIC en el personal docente comprenden un conjunto de actividades a desarrollar en los ambientes de aprendizajes las cuales, según Marqués (2000) comenta:

\begin{abstract}
Integración de recursos TIC (como instrumento, como recurso didáctico y como contenido de aprendizaje) en los planes docentes y programas formativos. Evaluación objetiva de recursos educativos en soporte TIC. Selección de recursos TIC y diseño de intervenciones formativas contextualizadas; organización de las clases. Aplicación en el aula de nuevas estrategias didácticas que aprovechen los recursos TIC: uso de las funcionalidades de la pizarra digital en el aula (ver http://dewey.uab.es/pmarques/pizarra.htm), realización de trabajos de autoaprendizaje a partir de búsquedas en Internet y presentación de los mismos en el aula con apoyos audiovisuales o digitales, realización de trabajos grupales en las aulas multiuso e informáticas, realización de proyectos colaborativos en soporte TIC a partir de las fuentes informativas de Internet y con la ayuda de los canales comunicativos telemáticos, utilización de las WebQuest y otros recursos del ciberespacio (ver http://dewey.uab.es/pmarques/buenaidea.htm), aprovechamiento didáctico de los recursos que proporcionan los "mass media"... Elaboración de apuntes, presentaciones y materiales didácticos multimedia de apoyo para los estudiantes. (p. 11)
\end{abstract}

\title{
Metodología
}

La investigación realizada es de tipo cualitativo basada en un estudio de caso, se observó el sujeto en estudio y se desarrolló una serie de fases: selección del caso, aplicación de un cuestionario, obtención de los datos y análisis de los datos. Básicamente se aplicó una encuesta para diagnosticar el nivel de conocimiento sobre las competencias tecnológicas digitales que poseen los facilitadores de la institución de educación universitaria seleccionada. La población estuvo conformada por un total de 149 docentes de las diferentes unidades curriculares, de la Universidad Nacional Experimental de la Seguridad (UNES), ubicada en el distrito capital, Los Flores de Catia, Caracas Venezuela. La muestra quedó constituida por 49 docentes, esto representa el $32 \%$ del total de la población.

El procedimiento desarrollado en la presente investigación consistió en el diseño de un instrumento de tipo encuesta disponible en Google Drive, validación de expertos, llenado de la encuesta por los participantes en línea, determinación de confiabilidad, tabulación de los datos, análisis e interpretación conclusiones y recomendaciones. En relación con el diseño del instrumento se utilizó un cuestionario con un total de 20 ítems, preguntas cerradas de tipo dicotómicas, respuestas múltiples y en la escala de Likert con cinco alternativas de respuestas: Muy de acuerdo, con un valor de 5 De acuerdo con un valor de 4, Ni de acuerdo con un valor de 3 , $\mathrm{Ni}$ en desacuerdo con un valor de 2 , En desacuerdo con un valor de 1 , Muy en desacuerdo con un valor de 0 puntos.

Se validó el instrumento mediante el juicio de tres expertos pertenecientes al área de Tecnología de la Información y la Comunicación. Mediante el coeficiente confiabilidad denominado Alpha de Cronbach dando como resultado 0,75 en la escala de magnitud lo cual es considerando de una confiabilidad muy alto, y en el cual la sumatoria de la varianza por cada pregunta dio como resultado 2,89 y la varianza total del instrumento dio como resultado 9,74.

\section{Análisis e interpretación de resultados}

Se obtuvo la muestra seleccionada y se basó en los resultados reflejados en los gráficos del cuestionario efectuado. Según los parámetros establecidos por el documento marco de la UNESCO titulado Estándares de Competencia en TIC para Docentes (UNESCO, 2008) Del cual se enfatizó tres dimensiones las cuales sintetizan los elementos fundamentales para el manejo de las competencias tecnológicas digitales por el personal docente, entre ellas se encuentran: dominio de conocimientos básicos digitales, dominio de competencias técnicas y actitud ante el uso de las TIC aplicadas en las prácticas docentes. $\mathrm{Al}$ respecto citan los autores Fernández, Leiva, y López (2017) 
Entre sus consideraciones está el hecho de poseer competencias digitales en relación con la profesión docente como algo imprescindible, así como las percepciones que señalan que la adquisición de las mismas para el alumnado resulta de gran importancia tanto para sus estudios y desempeño de funciones laborales futuras. (p. 9)

En relación con el dominio de conocimientos básicos digitales se obtuvo que la población encuestada en un 37,3\% según ítems $\mathrm{N}^{\circ} 1$ no han participado en talleres de alfabetización tecnológica, lo cual es contraproducente en la calidad de enseñanza facilitada por el personal docente, se evidencia qué no se está cumpliendo con lo planteado en el documento Estándares de competencias en TIC para Docentes (UNESCO, 2008): "Lograr la integración de las TIC en el aula dependerá de la capacidad de los maestros para estructurar el ambiente de aprendizaje de forma no tradicional, fusionar las TIC con nuevas pedagogías y fomentar las clases dinámicas" (p.7). Este enfoque teórico de generación de conocimientos compromete la capacidad de incrementar la productividad a lo largo de la vida, basado en la innovación y el aprendizaje permanente como objetivo político que va más allá del diseño curricular tradicional, propiciando las condiciones para la adquisición de nuevas habilidades entre ellas el pensamiento crítico, experimentación, colaboración, solución de problemas.

Entre los conocimientos elementales digitales se consideró evaluar mediante el Ítem $\mathbf{N}^{\circ} \mathbf{3}$ ¿Sabes qué es un software libre? Un porcentaje significativo $15,7 \%$ respondió de forma negativa factor que se suma a la necesidad de preparar a los docentes en el tema tecnológico. Por lo cual lo enunciado en el enfoque relativo a la generación de conocimiento en el material Estándares de Competencia en TIC para Docentes (UNESCO, 2008) , con el objetivo de formar trabajadores que se dediquen a la reproducción de conocimiento beneficiándose del mismo, se destaca lo siguiente "Describir la función y el propósito de las herramientas y recursos de producción de las TIC (equipos de grabación y producción multimedia, herramientas de edición, software para publicaciones y herramientas de diseño Web)". (p. 27)

En correspondencia a lo expuesto el docente entre sus competencias debe estar capacitado en el manejo del sitios Web y ubicación de software adecuados a los objetivos de aprendizaje, en este particular el material Estándares de Competencia en TIC para Docentes (UNESCO, 2008) cita: "Localizar paquetes de software educativo y recursos Web ya preparados, evaluarlos en función de su precisión y alineamiento con los estándares del plan de estudios (currículo), y adaptarlos a las necesidades de determinados estudiantes"(p.21) $\mathrm{Al}$ respecto se refleja lo siguiente en el Ítem $\mathrm{N}^{\circ} \mathbf{4}$ ¿Sabes que es un software educativo? un 25,5\% indico qué no, la cifra obtenida ratifica la debilidad evidente en competencias digitales que posee el personal en el uso de recursos pedagógicos digitales, situación que no se adecua a lo referido en el argumento teórico señalado anteriormente.

En este mismo orden de idea entre los resultados obtenidos se destacó la necesidad de evaluar el nivel del manejo integral de los docentes en los diferentes programas tecnológicos, en este punto se indagó en el Ítem $\mathrm{N}^{\circ} 10$ ¿Señale si ha utilizado los sistemas operativos? El mayor porcentaje se ubicó en el manejo de Windows con $64,7 \%$ y un $25 \%$ en el manejo de diferentes sistemas, de lo cual se deduce que un limitado número de docentes, posee conocimientos integrales de habilidades tecnológicas, incidiendo dicha situación en el nivel de desempeño en planificación educativa. La importancia de transformar esta realidad como tarea fundamental del personal docente para producción y profundización del conocimiento en la sociedad, esta es una de las premisas fundamentales expuestas en los Estándares de Competencia en TIC para Docentes (UNESCO, 2008) en el cuál se cita:

Los cambios educativos que guardan relación con la profundización del conocimiento pueden ser probablemente los mayores y tener más impacto en el aprendizaje. El objetivo de este enfoque en el plano de las políticas educativas consiste en aumentar la capacidad de educandos, ciudadanos y fuerza laboral para agregar valor a la sociedad y a la economía. (p. 8)

Comprender las nuevas tecnologías como objetivo de las políticas educativas para incrementar la productividad económica, el docente deberá tener la capacidad de conocer las características básicas del 
software de presentaciones multimedia y gráfico; en este contenido se exploró la información manejada por los docentes en el tema mediante la interrogantes planteadas en los Ítem N 12 ¿Sabes qué es una presentación electrónica? Un 9,8\% respondió de forma negativa, es de suma importancia que los docentes hagan uso de herramientas tecnológicas y se determinó que un porcentaje desconoce. Y el Ítem $\mathbf{N} \mathbf{1 8}$ en el cuál se interrogó ¿ Un multimedia es la combinación de las siguientes formas de contenido? Se obtuvo que un 96\% conoce que es un multimedia, no obstante un porcentaje pequeño de un $4 \%$ respondió de forma errónea, este promedio de docente no alcanza los estándares esperados, lo cual amerita de capacitación tecnológica. En estos contenidos basamentos teóricos del manejo de la información se destaca en Estándares de Competencia en TIC para Docentes (UNESCO, 2008) lo siguiente:

Examinar el objetivo del presentador multimedia y demostrar sus características generales y funcionamiento. Proponer a los participantes que elaboren, utilizando recursos informáticos, una presentación multimedia sobre un tema de su elección. Examinar el objetivo del software gráfico y mostrar cómo se crea una imagen. Solicitar a los participantes que creen visualizaciones gráficas y las intercambien. (p. 21)

Producir conocimiento aprendiendo de forma colaborativa proceso basado en el intercambio de saberes, visiones y conceptos que permitan aproximarse a la posible solución de problemas complejos de la realidad en la pedagogía del siglo XXI, exige un desarrollo gradual de las prácticas docentes integradas a factores positivos generados por el uso de la tecnología; al respecto en la segunda dimensión postura o, elemento de análisis incluido en Estándares de Competencia en TIC para Docentes (UNESCO, 2008) indica:

Los docentes tienen que estar en capacidad de diseñar comunidades de conocimiento basadas en las TIC, y también de saber utilizar estas tecnologías actitud ante el uso de las TIC para apoyar el desarrollo de las habilidades de los estudiantes tanto en materia de creación de conocimientos como para su aprendizaje permanente y reflexivo. (p. 17)

De acuerdo con el planteamiento teórico indicado en el párrafo anterior, en el análisis de resultados se enfocó una tríada de preguntas direccionadas en este particular, las cuales permiten observar la posición expresada al respecto por los docentes encuestados al respecto se obtuvo en el Ítem $\mathbf{N}^{\circ} \mathbf{5} \mathrm{El}$ uso de las herramientas TIC te permite: Actualización tecnológica, desarrollar estrategias pedagógicas novedosas, optimizar el proceso de aprendizaje, entre otros. Un 63,4\% estuvo muy de acuerdo, en contraste con el resto de los encuestados $31,7 \%$ señaló estar de acuerdo, cifra significativa la cual indica el interés un tanto

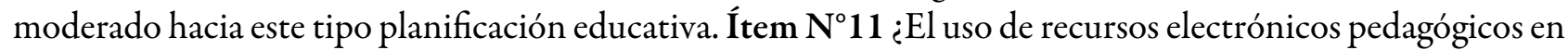
clase permite mejorar los procesos de aprendizaje? Un 31,7\% de los encuestados afirmó en estar de acuerdo, posición ante el uso de nuevos recursos tecnológicos, que se puede clasificar de aceptación mediana. Ítem $\mathbf{N}$ ${ }^{\circ} 15$ ¿Considera usted que las prácticas docentes deben apoyarse en el uso de las TIC? Un 47,9\% indicó en estar solo de acuerdo, cifra muy significativa, de lo cual se deduce que existe un elevado nivel de resistencia en la apropiación absoluta y global de los nuevos métodos de enseñanza.

En concordancia a la propuesta de investigación, los docentes necesitan empoderarse de herramientas digitales para facilitar a los estudiantes aprendizajes apoyados en las TIC, los recursos educativos que permitan enseñar las asignaturas integradas en nuevos conceptos y destrezas que construyan paradigmas de interés para el educando; entre las formas sofisticadas de diseñar el contenido educativo, se destacan los software educativo, con valor incalculable a la hora de efectuar simulaciones interactivas; al respecto se comenta en Estándares de Competencia en TIC para Docentes (UNESCO, 2008) "Manejar distintos programas no lineales de software que se adecuen a sus respectivas áreas académicas y que faciliten, por ejemplo, visualizaciones, análisis de datos, simulaciones de desempeño de funciones y referencias en línea”. (p. 24)

Según lo señalado en el párrafo anterior, se efectuó en el cuestionario dos preguntas para diagnosticar el uso de recursos digitales abiertos por parte de la población encuestada lo cual se ve reflejado en el Ítem $\mathrm{N}^{\circ} 16$ ¿Las presentaciones en Power Point permiten mostrar los contenidos a los estudiantes de forma atractiva? 43,1\% indicó en estar de acuerdo, es de considerar en el uso de este recurso cierto nivel de resistencia, igualmente 
un porcentaje pequeño muestra un nivel de indiferencia al indicar ni de acuerdo, ni en desacuerdo. Y el Ítem $\mathrm{N}^{\circ} 17$ ¿El software educativo es una herramienta pedagógica que ayuda a la adquisición de conocimientos y al desarrollo de habilidades? Un 6\% se ubicó en una posición neutral, igualmente un $48 \%$ afirmó en estar de acuerdo, estas dos categorías suman un 54\% se interpreta de dichas cifras un grado elevado de no adaptación y posible rechazo al uso de las tecnologías educativas. El uso de esta estrategia educativa se relaciona a lo que se señala en el documento Estándares de competencia en TIC para Docentes (UNESCO, 2008) el cual cita:

Gracias a la utilización continua y eficaz de las TIC en procesos educativos, los estudiantes tienen la oportunidad de adquirir capacidades importantes en el uso de estas. El docente es la persona que desempeña el papel más importante en la tarea de ayudar a los estudiantes a adquirir esas capacidades. Además, es el responsable de diseñar tanto oportunidades de aprendizaje como el entorno propicio en el aula que faciliten el uso de las TIC por parte de los estudiantes para aprender y comunicar. Por esto, es fundamental que todos los docentes estén preparados para ofrecer esas oportunidades a sus estudiantes. (p. 2)

En continuidad con el tema en los resultados obtenidos y su concordancia con los argumentos teóricos Estándares de Competencia en TIC para Docentes (UNESCO, 2008) se establece la importancia de disponer de una computadora para su adecuado desempeño laboral se indica: "Alfabetismo en TIC". Las repercusiones de este enfoque para la formación de docentes son, principalmente, fomentar el desarrollo de habilidades básicas en las TIC y la utilización de estas para el mejoramiento profesional” (p.15).Por ende otro elemento a considerar es el acceso a la computadora como una herramienta indispensable, para lo cual se efectuó en el Ítem $\mathrm{N}^{\circ} 2$ la siguiente interrogante: ¿Posee computadora? Al respecto un 95\% respondió de forma positiva, lo que se considera favorable, no obstante el $5 \%$ restante se encuentra en desventaja y a su vez representa una seria limitante para la práctica docente.

En concordancia con el tema en estudio en relación con los resultados obtenidos en competencias técnicas. Se efectuó dos interrogantes: Ítem 6 ¿Alguna vez has participado en un foro digital? 86,3\% respondió afirmativo, no obstante un $13,7 \%$ respondió negativo demostrando así las deficiencias en este orden, la destreza en saber participar en foros digitales es indispensable hoy en día en el campo docente. Ítem 7¿ Utiliza recursos electrónicos en clase? un $94,1 \%$ respondió positivo, sin embargo un $6 \%$ no están formados en el tema. Como se pudo observar un porcentaje de los encuestados presentan deficiencias en el manejo técnico de las herramientas digitales mencionada. En este punto la comunicación basada en el uso de videoconferencias y sus distintas modalidades mensajes de texto, recursos electrónicos, aprendizaje colaborativo mediante el uso de internet para el diseño de estrategias pedagógicos y actividades en línea que permitan resolver problemas e investigar desde la participación de equipos colaborativos en chat o foro virtual; permite hacer referencia al enfoque generación de conocimiento, en cual plantea como objetivo político aumentar la productividad y la capacidad de solucionar problemas desde la innovación según lo planteado en el documento titulado Estándares de Competencia en TIC para Docentes (UNESCO, 2008) en el cual se cita: "Diseñar materiales y actividades en línea que comprometan a los estudiantes en la solución de problemas, la realización de trabajos, la investigación o la creación artística, de manera colaborativa”. (p. 26)

En este mismo orden de ideas entre los resultados obtenido se indagó mediante dos interrogantes el manejo básico de competencias TIC, Ítem 8 ¿Señale si ha trabajado en las herramientas de oficina de Microsoft? un $80,4 \%$ han trabajado en variadas herramientas de Microsoft, no obstante se destaca que 5,9\% solo ha trabajado en Word. Ítem 9 ¿Señale si ha trabajado en las herramientas del libro office? un 31,4\% no conoce las herramientas de Libreoffice, dicha competencia es de suma importancia en el ejercicio de la docencia, considerando las ventajas del manejo apropiado del software libre. En este aspecto técnico el documento referido en oportunidades anteriores ratifica el argumento teórico de los objetivos educativos de formar docentes con capacidad de incrementar la productividad económica al conocer, comprender y manejar las nuevas tecnologías Estándares de Competencia en TIC para Docentes (UNESCO, 2008) cita: "el uso de hardware corriente Examinar y demostrar el funcionamiento del hardware más básico: computadores de escritorio (PC), portátiles y de mano (tipo Palm);impresoras y escáneres. Utilizaciones básicas de procesadores de texto tales como digitación, edición, impresión de textos”. (p. 20) 
Por otra parte los resultados obtenidos nos permiten observar en el tema del uso de recursos digitales el nivel en el cual se ubica la población encuestada, al respecto se destaca la importancia y valor en la profundización del conocimiento mediante estas herramienta, cabe destacar que el docente debe estar comprometido con las prácticas docentes basadas en saber utilizar los recursos digitales, de acuerdo con las premisas teóricas del documento base del 2008 mencionado en oportunidades anteriores, el paradigma político educativo del siglo XXI permite afianzar y avanzar en la profundización del conocimientos estimulando en la sociedad la capacidad de abordar y buscar soluciones a los problemas contemporáneos. En este objetivo en Estándares de Competencia en TIC para Docentes (UNESCO, 2008). Al respecto se cita: "Identificar o concebir problemas complejos del mundo real y estructurarlos de manera que integren conceptos esenciales de los contenidos y sirvan de base para los proyectos de los estudiantes" (p.23).

En este mismo orden de ideas los recursos de apoyo para la innovación educativa, amerita de examinar y evaluar las prácticas docentes direccionadas en la apropiación del uso oportuno y competente de las TIC que garanticen el aprovechamiento de diseños de materiales educativos digitales atractivos y novedosos. En este particular en el material consultado titulado Estándares de Competencia en TIC para Docentes (UNESCO, 2008) se cita

Mostrar la utilización de software de presentación multimedia y otros recursos informáticos para complementar un curso magistral; suministrar una serie de ejemplos de presentaciones multimedia educativas; solicitar a los participantes que elaboren un proyecto de clase que incluya la utilización del presentador multimedia; y pedirles que utilicen este software para preparar una presentación. (p. 20)

Según el análisis obtenido y en concordancia con el párrafo anterior el elemento conceptual del significado positivo del aprendizaje creativo se destacan alternativas educativas, como lo son el uso de las presentaciones digitales multimedia y gráficas, en este objetivo se consideró de importancia reflejar entre los resultados el uso de dichas herramientas pedagógicas por parte de la población en estudio mediante una serie de interrogantes entre las cuales se destacan: Ítem 13.¿Has utilizado presentaciones electrónicas como apoyo pedagógico? De los encuestados un $91,8 \%$ respondió de forma positiva, sin embargo un 7,8\% respondió negativo, porcentaje que amerita de actualización tecnológica. Ítem 14. ¿Cuál de los siguientes programas has utilizado para elaborar presentaciones electrónicas como recursos pedagógicos? Se destacó el Power Point entre las herramientas más usadas para presentaciones electrónicas totalizándose un 79,6\% de preferencia hacia este programa, lo cual se considera como una desventaja ya que de cierto modo se encuentra obsoleto ante las nuevas tendencias; en Prezi se obtuvo 11,8\% y un 5,9\% ninguna; Ítem 19¿Cuáles de los siguientes recursos pedagógicos utiliza con mayor frecuencia? $41,2 \%$ indicó videos, el uso mayoritario de este recurso como estrategia pedagógica basadas en material prediseñado como único punto de apoyo en clase, se le puede considerar como una actividad educativa de reforzamiento, más no complementa la totalidad del proceso de aprendizaje, también se evidenció que un porcentaje considerable de 11,8\% indicó la práctica tradicional del uso de material diseñado en papel bond, lo qué significa una gran desventaja ante la necesidad de incorporar métodos interactivos de estímulo e interés en la participación activa de los estudiantes,

Otro de los elementos tomados en cuenta en el estudio efectuado y el análisis de sus resultados es la relación de las políticas educativas orientadas a la obtención de conocimientos tecnológicos, mediante la formación profesional de los docentes, lo cual implica poner al alcance del personal recursos pedagógicos de calidad, incrementando la adquisición de las nociones básicas del manejo de herramientas digitales entre ellas hardware y software, lo cual amerita de una transformación profunda en las prácticas docentes tradicionales; generando el uso de tecnologías digitales múltiples en las actividades académicas individuales o grupales. Se puede observar al respecto en el Ítem 20 ¿Cómo considera usted su nivel de manejo en las TIC? Un 41,2\% consideró que se ubican en regular, dicha cifra no concuerda con las competencias tecnológicas digitales que debe manejar el docente; debe estar ajustado al perfil según los parámetros establecidos en el documento titulado Estándares de Competencia en TIC para Docentes (UNESCO, 2008) de lo contrario se pone en riesgo la calidad educativa. En el documento mencionado se cita: 
Los programas de formación de docentes deberían coordinar las competencias profesionales del profesorado, cada vez más complejas, haciendo uso generalizado de las TIC para apoyar a los estudiantes que crean productos de conocimiento y que están dedicados a planificar y gestionar sus propios objetivos y actividades. Esto debe realizarse en una escuela que, de por sí, sea una organización que aprende y mejora continuamente. En este contexto, los docentes modelan el proceso de aprendizaje para los alumnos y sirven de modelo de educando, gracias a su formación profesional permanente (individual y colaborativamente). En este caso, la escuela fomenta el desarrollo de la sociedad del conocimiento contemplada por la Comisión Internacional de la Educación para el Siglo XXI. (p. 8)

\section{Figura 1: ¿Has participado en talleres de iniciación informática?}

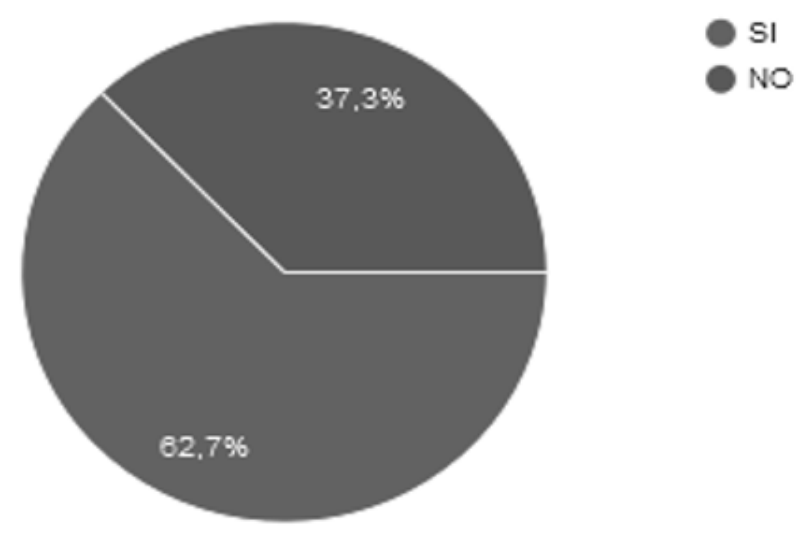

Fuente: Elaboración propia

FIGURA 1

¿Has participado en talleres de iniciación informática?

Fuente: Elaboración propia

\section{Figura 2: ¿Posee computadora?}

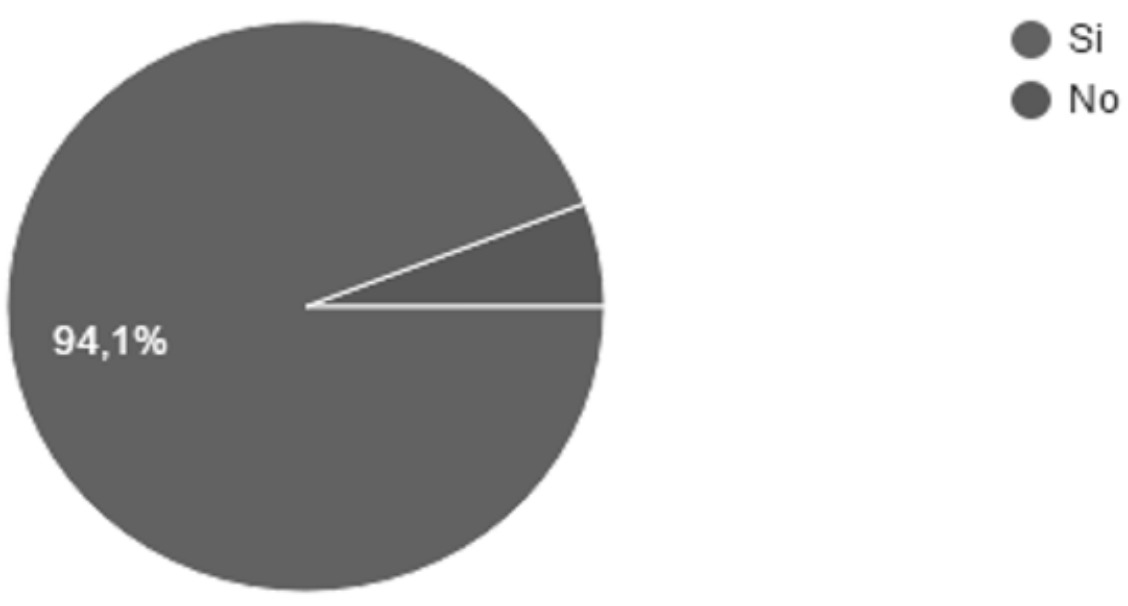

Fuente: Elaboración propia

FIGURA 2

¿Posee computadora?

Fuente: Elaboración propia 
Figura 3: ¿Sabes qué es un software libre?

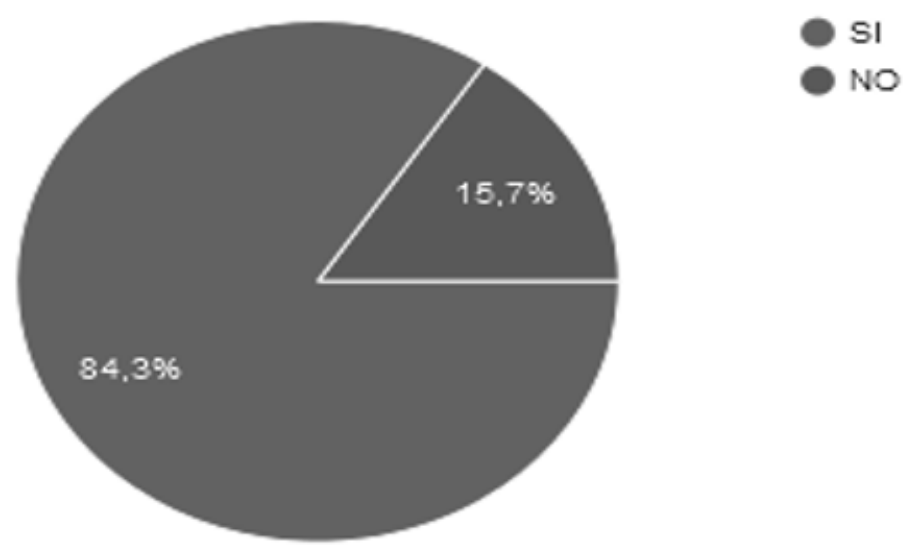

Fuente: Elaboración propia

FIGURA 3

¿Sabes qué es un software libre?

Fuente: Elaboración propia

Figura 4: ¿Sabes qué es un software educativo?

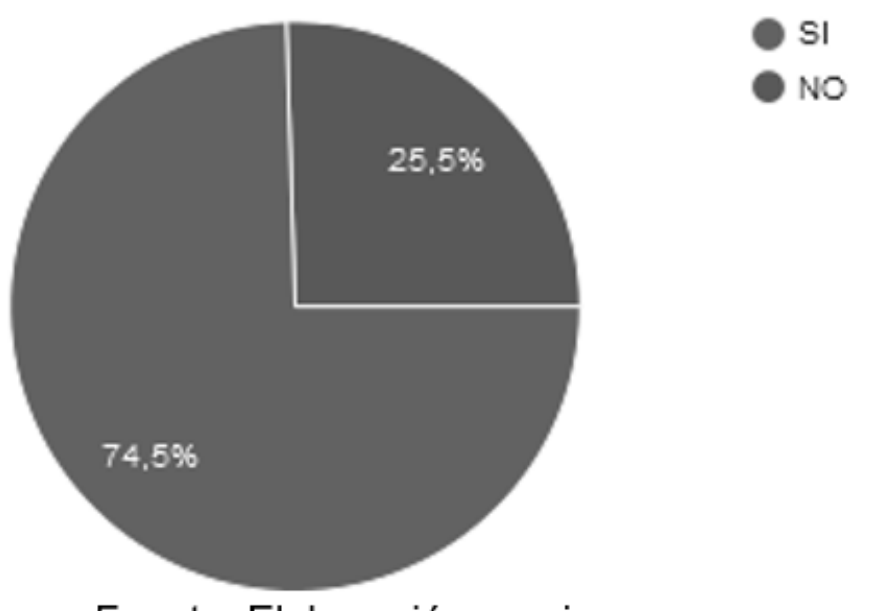

Fuente: Elaboración propia

FIGURA 4

¿Sabes qué es un software educativo?

Fuente: Elaboración propia 
Figura 5: El uso de las herramientas TIC te permite: Actualización tecnológica, desarrollar estrategias pedagógicas novedosas, optimizar el proceso de aprendizaje, entre otros.
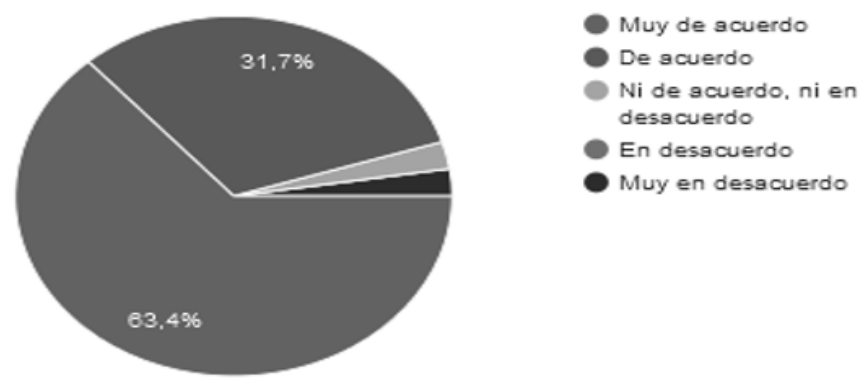

Fuente: Elaboración propia

FIGURA 5

El uso de las herramientas TIC te permite: Actualización tecnológica, desarrollar estrategias pedagógicas novedosas, optimizar el proceso de aprendizaje, entre otros. Fuente: Elaboración propia

Figura 6: ¿Alguna vez has participado en un foro digital?

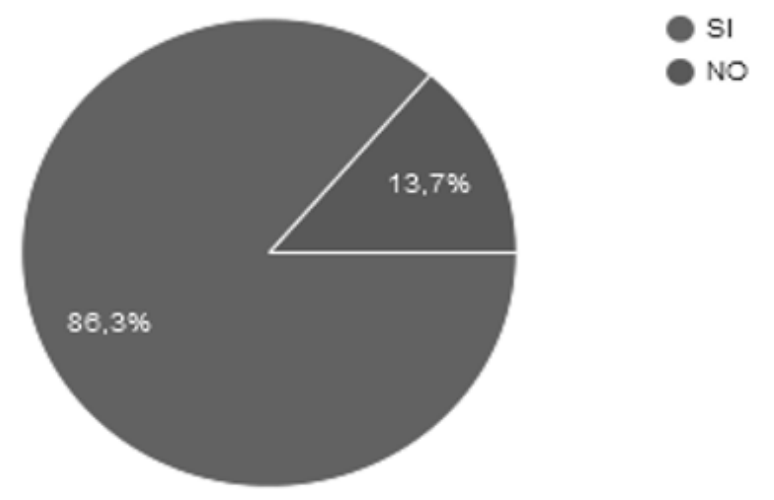

Fuente: Elaboración propia

FIGURA 6

¿Alguna vez has participado en un foro digital?

Fuente: Elaboración propia 


\section{Figura 7. ¿Utiliza recursos tecnológicos en clase?}

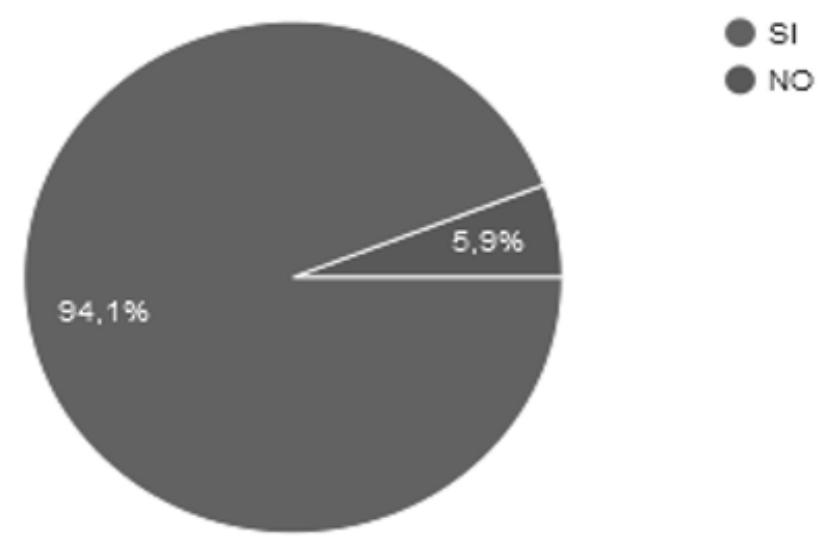

Fuente: Elaboración propia

FIGURA 7

¿Utiliza recursos tecnológicos en clase?

Fuente: Elaboración propia

Figura 8. ¿Señale si ha trabajado en las siguientes herramientas de oficina de Microsoft?

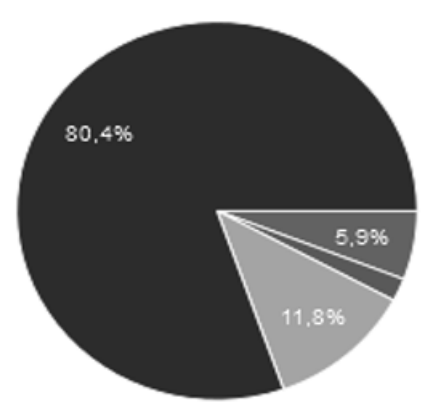

Word (Procesador de

Texto)

- Excel (Hoja de Calculo)

Powert Point

Presentación electrónica)

Ninguna de las anteriores

- Todas las anteriores

Fuente: Elaboración propia

FIGURA 8

¿Señale si ha trabajado en las siguientes herramientas de oficina de Microsoft?

Fuente: Elaboración propia 
Figura 9: ¿Señale si ha trabajado en las siguientes herramientas de Libreoffice?

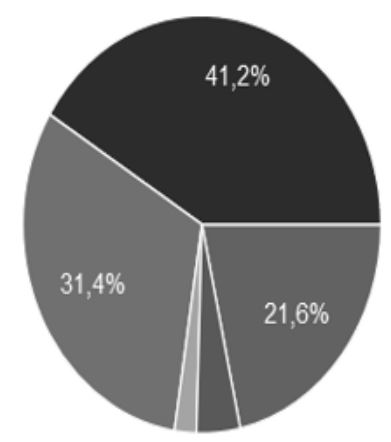

Writer ( Procesador de Texto)

Calc (Hoja de Calculo)

Impress (Presentación electrónica)

Ninguna de las anteriores

Todas las anteriores

\section{Fuente: Elaboración propia}

FIGURA 9

¿Señale si ha trabajado en las siguientes herramientas de Libreoffice?

Fuente: Elaboración propia

Figura 10: ¿Señale si ha utilizado los siguientes sistemas operativos?

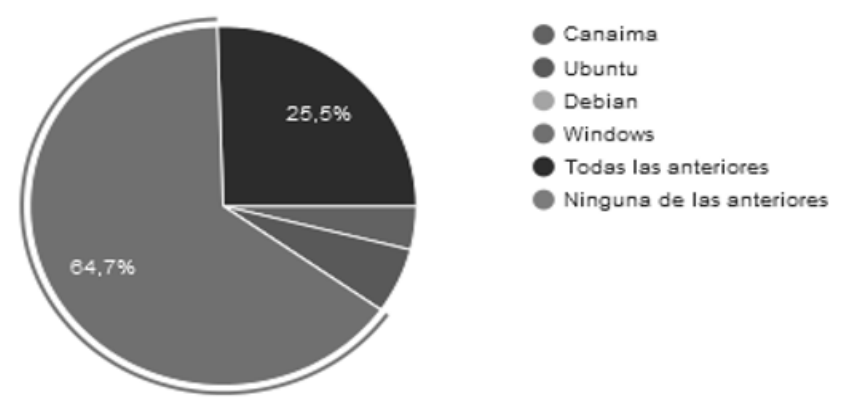

Fuente: Elaboración propia

FIGURA 10

¿Señale si ha utilizado los siguientes sistemas operativos?

Fuente: Elaboración propia 
Figura 11: ¿El uso de recursos electrónicos pedagógicos en clase permite mejorar los procesos de aprendizaje?

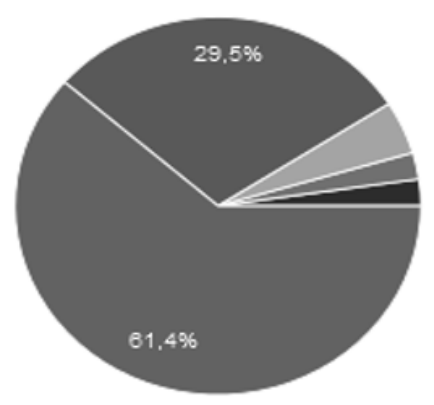

Muy de scuerdo

De acuerdo

Ni de scuerdo, ni en

desscuerdo

- En desacuerdo

- Muy en desscuerdo

Fuente: Elaboración propia

FIGURA 11

¿El uso de recursos electrónicos pedagógicos en clase permite mejorar los procesos de aprendizaje? Fuente: Elaboración propia

\section{Figura 12: ¿Sabes qué es una presentación electrónica}

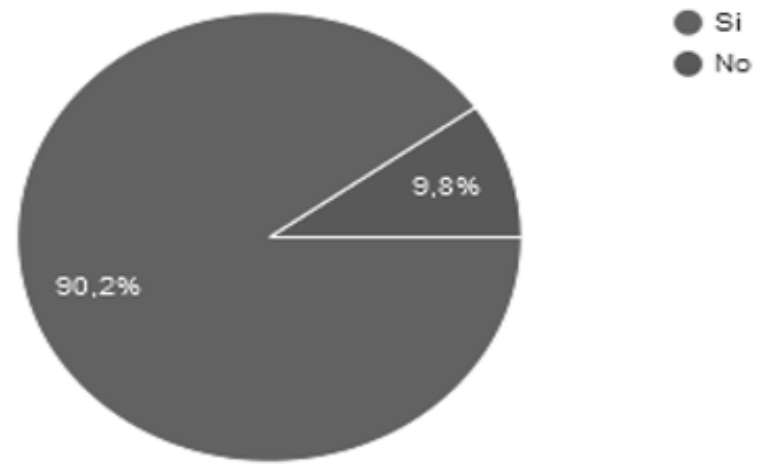

Fuente: Elaboración propia

FIGURA 12

¿Sabes qué es una presentación electrónica?

Fuente: Elaboración propia 
Figura 13: ¿Has utilizado presentaciones electrónicas como apoyo pedagógico?

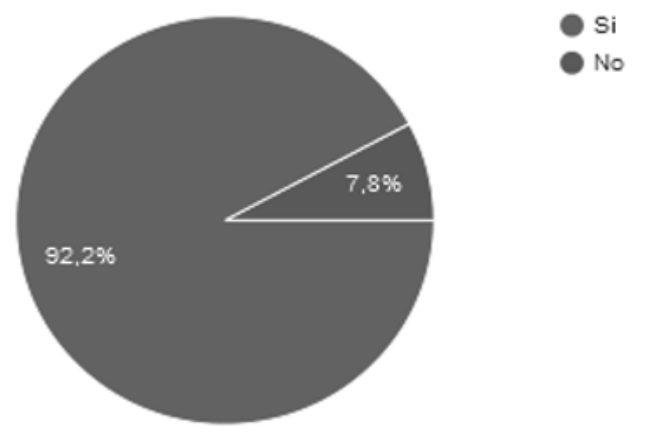

Fuente: Elaboración propia

FIGURA 13

¿Has utilizado presentaciones electrónicas como apoyo pedagógico? Fuente: Elaboración propia

Figura 14: ¿Cuáles de los siguientes programas has utilizado para elaborar presentaciones electrónicas como recursos pedagógicos?

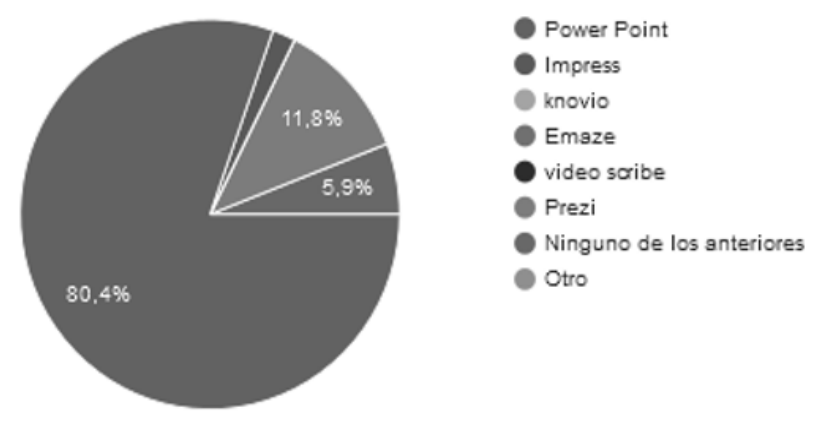

Fuente: Elaboración propia

FIGURA 14

¿Cuáles de los siguientes programas has utilizado para elaborar presentaciones electrónicas como recursos pedagógicos?

Fuente: Elaboración propia 
Figura 15: ¿Considera usted que las prácticas docentes deben apoyarse en el uso de las TIC?
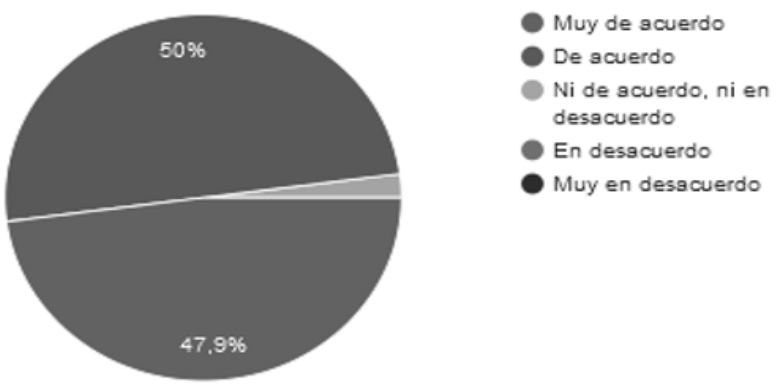

Fuente: Elaboración propia

FIGURA 15

¿Considera usted que las prácticas docentes deben apoyarse en el uso de las TIC?

Fuente: Elaboración propia

Figura 16: ¿Las presentaciones en Power Point permiten mostrar los contenidos a los estudiantes de forma atractiva?
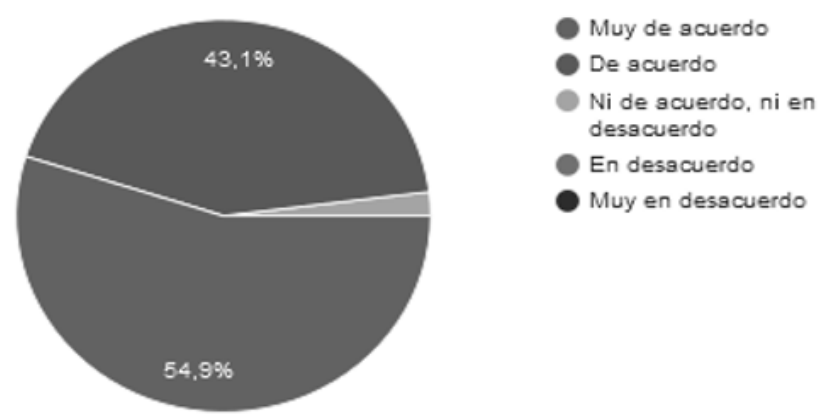

Fuente: Elaboración propia

FIGURA 16

¿Las presentaciones en Power Point permiten mostrar los contenidos a los estudiantes de forma atractiva? Fuente: Elaboración propia

Figura 17: ¿El software educativo es una herramienta pedagógica que ayuda a la adquisición de conocimientos y al desarrollo de habilidades?
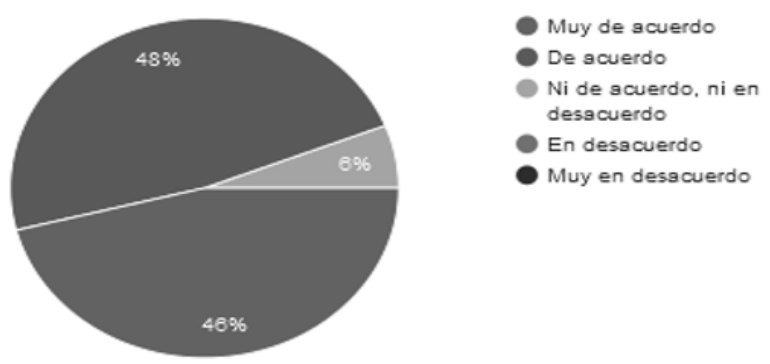

Fuente: Elaboración propia

FIGURA 17

¿El software educativo es una herramienta pedagógica que ayuda a la adquisición de conocimientos y al desarrollo de habilidades? Fuente: Elaboración propia 
Figura 18: ¿Un multimedia es la combinación de las siguientes formas de contenido?

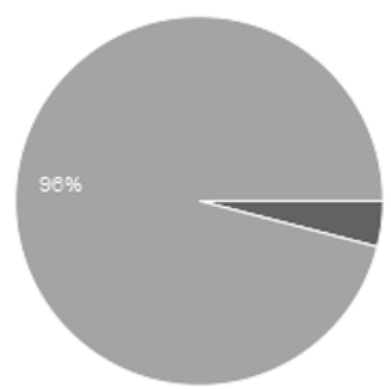

imsgén

Música y texto

Texto,sonido imagen,

vídeo, snimación

Fuente: Elaboración propia

FIGURA 18

¿Un multimedia es la combinación de las siguientes formas de contenido? Fuente: Elaboración propia

Figura 19: ¿Cuál de los siguientes recursos pedagógicos utilizas con mayor frecuencia?

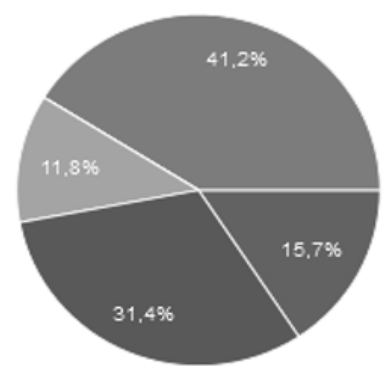

videos

presentaciones electrónicas

material impreso/ o diseños en papel bond

audios

- Ninguno de los anteriores

- Todas las anteriores

Fuente: Elaboración propia

FIGURA 19

¿Cuál de los siguientes recursos pedagógicos utilizas con mayor frecuencia? Fuente: Elaboración propia

Figura 20: ¿Cómo considera usted su nivel de manejo de las TIC?

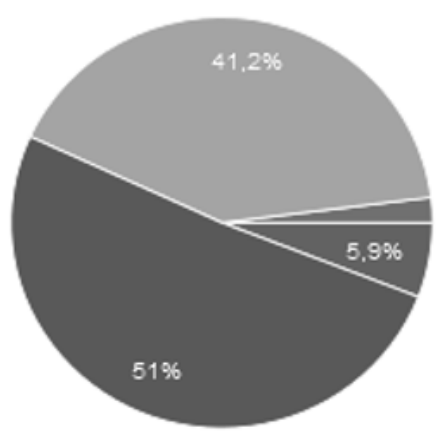

$$
\begin{aligned}
& \text { Muy bueno } \\
& \text { Bueno } \\
& \text { Regular } \\
& \text { Deficiente } \\
& \text { Muy deficiente }
\end{aligned}
$$

Fuente: Elaboración propia

FIGURA 20

¿Cómo considera usted su nivel de manejo de las TIC?

Fuente: Elaboración propia 


\section{ReFERENCIAS}

Adell, J. (1997). Tendencias en Educación en la Sociedad de las Tecnologías de la Información. Revista EDUTEC, 7. Recuperado de http://nti.uji.es/docs/nti/Jordi_Adell_EDUTEC.html

Arrufat, M. y Sánchez, V. (2010). El Futuro Docente Ante Las Competencias En El Uso De Las Tecnologías De La Información y Comunicación Para Enseñar. EDUTEC. Revista Electrónica de Tecnología Educativa. 34. Recuperado de http://www.edutec.es/revista/index.php/edutec-e/article/view/

Barroso, J. (2007). La alfabetización tecnológica. En Cabero, J. (coord.) Tecnología educativa (pp. 92- 123). Madrid: Mc Graw Hill.

Cabero, J. y Romero, R., (2010). Diseño y Producción de TIC para la formación.. Recuperado de https:// books.google.co.ve/books?isbn $=849788163 \mathrm{X}$

Cacheiro, M. (2014). Educación y Tecnología: Estrategias Didácticas para la Integración de las TIC. Recuperado de https://books.google.co.ve/books?id=8fylAwAAQBAJ\&

Fernández, E., Leiva, J. y López, E. (2017). Competencias digitales en docentes de Educación Superior. Revista Digital de Investigación en Docencia Universitaria, 12(1), 213-231. Recuperado de https://revistas.upc.edu.pe/ index.php/docencia/article/view/558/752

Heras, V., Roa, R. y Espinoza, A. (2015). Las Competencias Digitales de Futuros Docentes y su Relación con los Estándares Internacionales en TIC'S. Revista Iberoamericana de Producción Académica y Gestión Educativa. Recuperado de https://bit.ly/2Qrt4Qd

Gallego, M., Gámiz, V. y Gutiérrez, E. (2010). El futuro docente ante las competencias en el uso de las tecnologías de la información y comunicación para enseñar. EDUTEC. Revista Electrónica de Tecnología Educativa, 34. Recuperado de http://edutec.rediris.es/revelec2/revelec34/

González, J. (2018). Uso de la Tecnología en la Educación. México, UNID editorial digital 31. Recuperado en https:// bit.ly/2SPRxQw

González, J. y Wagenaar, R. (2003). Tuning Educational Structures in Europe. Informe Final - Proyecto Piloto, Fase 1. Bilbao: Universidad de Deusto

Gómez, W. (2010). Significado que le dan los profesores al uso de las tics en los procesos de enseñanza y de aprendizaje en dos instituciones educativas. Recuperado de http://www.colombiaaprende.edu.co/html/docentes/

Gros, B., Silva, J., Garrido, J. y Rodríguez, J. (2006). Estándares en Tecnología de la Información y la Comunicación para la Formación Inicial Docente - Situación actual y el caso chileno. Revista Iberoamericana de Educación, 38(1), 51-68. Recuperado de http://www.rieoei.org/1391.htm

Mirabal, A (2013). Competencias Tecnológicas Clave para el Uso de Moodle como Apoyo a la Docencia Presencial Universitaria. (Tesis de maestría). Tecnológico de Monterrey, República Dominicana. Recuperado de https:// goo.gl $/ \mathrm{n} 7 \mathrm{hLYo}$

Marqués, P. (2000). Los Docentes: Funciones, Roles, Competencias Necesarias. Recuperado de https:// bit.ly/1PsQf79

Tejada, J. (2009). Competencias docentes. Profesorado. Revista de Currículum y Formación de Profesorado, 2. Recuperado de http://www.redalyc.org/pdf/567/56711798015.pdf

UNESCO (2008). Estándares de competencia en TIC para docentes. Londres. Recuperado de http:// eduteka.icesi.edu.co/pdfdir/UNESCOEstandaresDocentes.pdf

Sánchez, M. y Talavera, L. (2008). Pautas para la elaboración de la programación didáctica. Caracas: Universidad Iberoamericana del Deporte. Recuperado de: http://www.uideporte.edu.ve/WEB/pdf/PAUTA

Páez, H. (2008). Alfabetización digital para docentes de postgrado. PARADIGMA, Revista Electrónica del CIEP Centro de Investigaciones Educacionales. 2, (9). Recuperado de http://revistas.upel.edu.ve/index.php/ paradigma/issue/view/448.

\section{BY-NC-ND}

